\title{
Variation in guided streamer propagation along a DBD plasma jet by tailoring the applied voltage waveform
}

Cite as: Appl. Phys. Lett. 116, 164102 (2020); https://doi.org/10.1063/5.0006178

Submitted: 28 February 2020 . Accepted: 05 April 2020 . Published Online: 20 April 2020

M. E. Pinchuk (D) O. M. Stepanova (D), M. Gromov (D), Ch. Leys, and A. Nikiforov (D)

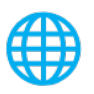

\section{Hall Effect} Measurement Handbook

A comprehensive resource for researchers Explore theory, methods, sources of errors, and ways to minimize the effects of errors
Request it here

Alakeshore 


\title{
Variation in guided streamer propagation along a DBD plasma jet by tailoring the applied voltage waveform
}

Cite as: Appl. Phys. Lett. 116, 164102 (2020); doi: 10.1063/5.0006178

Submitted: 28 February 2020 - Accepted: 5 April 2020 .

Published Online: 20 April 2020

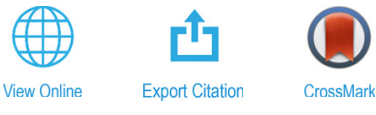

M. E. Pinchuk, ${ }^{1, a)}$ (D) O. M. Stepanova, ${ }^{1}$ (D M. Gromov, ${ }^{2,3}$ (D) Ch. Leys, ${ }^{2}$ and A. Nikiforov ${ }^{2}$

\begin{abstract}
AFFILIATIONS
${ }^{7}$ Institute for Electrophysics and Electrical Power of the Russian Academy of Sciences, Dvortsovaya Naberezhnaya 18, Saint-Petersburg 191186, Russia

${ }^{2}$ Department of Applied Physics, Ghent University, Sint-Pietersnieuwstraat 41, Gent 9000, Belgium

${ }^{3}$ Chimie des Interactions Plasma-Surface (ChIPS), CIRMAP, Universite de Mons, 20 Place du Parc, B-7000 Mons, Belgium
\end{abstract}

${ }^{\text {a) }}$ Author to whom correspondence should be addressed: pinchme@mail.ru

\begin{abstract}
Experimental data on the evolution of a helium atmospheric pressure plasma jet driven by two different voltage waveforms are presented. The characteristics of directed ionization waves (guided streamers) were compared for a sinusoidal voltage waveform with a frequency of $52 \mathrm{kHz}$ and a voltage waveform that was formed via the superposition of $41.6 \mathrm{kHz}$ bipolar square pulses and $300 \mathrm{kHz}$ oscillations. With the sinusoidal voltage, two consecutive ionization waves were observed. With a special tailoring voltage, control of the guided streamer propagation in a stepwise mode was achieved. The observed second streamer and the second step of propagation could be regarded as a secondary ionization wave for both voltages. A change in the voltage waveform led to significant variations in the secondary ionization wave formation and streamer parameters. The voltage waveform enabled the number of ionization waves and their propagation to change, which provided the possibility of controlling the plasma parameters of the jet.
\end{abstract}

Published under license by AIP Publishing. https://doi.org/10.1063/5.0006178

Atmospheric pressure plasma jets (APPJs) have been extensively studied in recent years, which is mostly due to their application prospects in life sciences. ${ }^{1-4}$ Widely used plasma sources for producing APPJs are often based on a dielectric barrier discharge (DBD) that is operated with a noble gas flow passing through a discharge gap.

It has been demonstrated in many studies that a continuous luminous APPJ is a sequence of directed ionization waves (IWs), which are often called guided streamers, ${ }^{5-7}$ and is visible as a highvelocity radiated light area, which is called a "plasma bullet." 8 The head of a guided streamer is a highly effective chemical reactor that can produce active species for various applications. ${ }^{9,10}$ In addition, an APPJ can transfer high electric fields across substantial distances ${ }^{6,11-13}$ and deposit charge on remote objects. ${ }^{10,11}$ One of the main APPJ applications is for the local modification of complex 2D or 3D targets, including living tissue surfaces. ${ }^{10,11}$ Such interaction of highly energetic plasma and living matter has attracted substantial scientific and practical interest, but many relevant questions remain to be answered. ${ }^{2,3,14,15}$
First, a problem arises in controlling the jet propagation. As a strategy for the realization of guided streamer control, the application of an external electric field has been investigated. ${ }^{12,16-18}$ It has been shown that an electric potential that is applied to an external additional electrode or treated target changes the behavior of the streamer propagation and its velocity along the APPJ.

Another strategy that is under consideration for controlling the propagation of the streamer is the modification of an applied voltage waveform. ${ }^{19}$ For example, up to five consecutive bullets were recorded for a positive half-period in a helium APPJ, which corresponded to current pulses in the discharge gap, for a frequency of $10 \mathrm{kHz}$ in Ref. 20. The voltage decrease and increase in the frequency to $50 \mathrm{kHz}$ resulted in a reduction of the number of bullets to one, ${ }^{21}$ which corresponded to one current pulse. Indeed, current pulses in a DBD can be controlled via voltage tailoring, as was demonstrated in numerical calculations. $^{22}$

For APPJ control, a more promising approach is the active management of the applied voltage. Via the use of the methods that are 
discussed above, periodically swelling structures with a period that is proportional to the bias voltage frequency were obtained for an argon APPJ. ${ }^{23}$ For a helium APPJ, a stepwise propagation of a guided streamer was observed in our recent works. ${ }^{24,25}$ This phenomenon was realized with a power supply that generated an output voltage signal that consisted of a superposition of microsecond bipolar square pulses and oscillating signals. The numerical calculations ${ }^{26}$ showed the possibility of a stepwise scenario for streamer propagation with the corresponding modulation of the nanosecond-pulsed applied voltage.

The objective of this study was to demonstrate the control of the streamer dynamics in a system via voltage waveform changes. Here, we analyzed data for two types of applied voltage waveforms with similar periods with an emphasis on the effect of the tailored voltage waveform on the guided streamer properties in a helium APPJ. The first voltage waveform was a "classical" sinusoidal signal with a frequency of $52 \mathrm{kHz}$, whereas the second tailored voltage signal consisted of a superposition of $41.6-\mathrm{kHz}$ bipolar square pulses and a $300-\mathrm{kHz}$ oscillating high-voltage signal. To compare the two regimes, the same peak-to-peak voltage of $4.6 \mathrm{kV}$ was fixed throughout the experiments. Electrical parameters for both the regimes are presented in Fig. 1.

The voltage across the gas discharge unit and the charge through the discharge circuit were measured using a Tektronix P6015A highvoltage probe and a charge monitor with a capacitance of $59 \mathrm{pF}$.
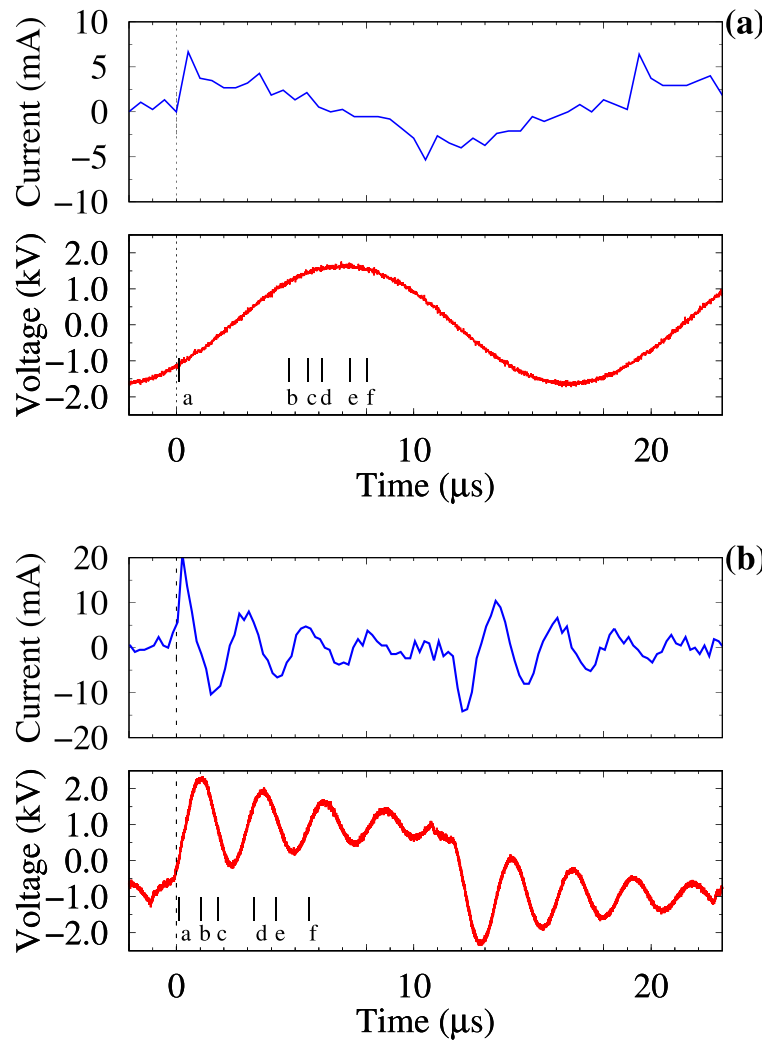

FIG. 1. Current on the discharge electrode and high-voltage signal: a sinusoidal waveform (a) and a superposition of bipolar square pulses and oscillating signals (b). The labels $(\mathrm{a})-(\mathrm{f})$ are frame times that correspond to the frame labels in Figs. 3 and 4.
All signals were recorded using a LeCroy WS64Xs (Teledyne LeCroy, USA; bandpass $600 \mathrm{MHz}$ ) oscilloscope. The current curves were obtained by differentiating the charge waveform that was recorded by the charge monitor.

An APPJ that was directed vertically upward with a jet configuration of "inner central rode-gas gap-quartz tube (dielectric barrier)-outer ring" was investigated, which was similar to the APPJ that was used in Ref. 27. A high voltage was applied to the copper inner electrode, which had a diameter of $1.5 \mathrm{~mm}$, and the outer grounded electrode that was constructed from 5 -mm-thick copper foil that was positioned $5 \mathrm{~mm}$ from the jet edge. The quartz tube had an internal diameter of $4.6 \mathrm{~mm}$ and a wall thickness of $1 \mathrm{~mm}$.

The He flow rate, which was controlled by a 1179 Mass-Flo flow controller (MKS Instruments, USA), was set at $5.81 / \mathrm{min}$. The value of the gas flow was selected such that the flow was laminar and the length of the plasma jet was maximal..$^{24,25,28}$ The jet was sustained in ambient air at an air temperature of $20^{\circ} \mathrm{C}$ and a relative humidity of $48 \%$.

High-speed imaging was conducted using a Hamamatsu C8484-05G charge-coupled device camera with a W7571-01 image intensifier unit (Hamamatsu Photonics K.K., Japan) and an exposure of $50 \mathrm{~ns}$.

Molecular bands of $\mathrm{N}_{2}, \mathrm{~N}_{2}^{+}$, and $\mathrm{OH}$ and oxygen, hydrogen, and helium lines were observed in the APPJ integral spectrum in the range of 300-800 nm (Fig. 2). The $\mathrm{N}_{2}$ and $\mathrm{N}_{2}^{+}$bands were of the highest intensity in the plasma effluent. In the range of wavelengths above $500 \mathrm{~nm}$, only an intense He line at $706.5 \mathrm{~nm}$ was observed. A weak emission line of $\mathrm{O}$ I at $777.8 \mathrm{~nm}$ was observed, which was of negligible intensity; therefore, it was excluded from the analysis. The spectra have been recorded using an S2000 spectrometer (Ocean Optics, USA; sensitivity range $220-925 \mathrm{~nm}$, entrance slit $10 \mu \mathrm{m}, 360 \mathrm{~g} / \mathrm{mm}$ grating blazed at $500 \mathrm{~nm}$ with a resolution of $1.3 \mathrm{~nm}$ ). UV enhanced fiber optics of $600 \mu \mathrm{m}$ diameter with a collimating lens has been connected to the spectrometer for light collection.

So imaging was conducted with optical filters for three cases (see Fig. 2): imaging without spectral filtration [(a1)-(f1) in Figs. 3 and 4], which corresponded to the camera spectral range of $300-800 \mathrm{~nm}$; imaging with an optical filter with a transmittance band of 500-800 nm [(a2)-(f2) in Figs. 3 and 4], which corresponded to He I

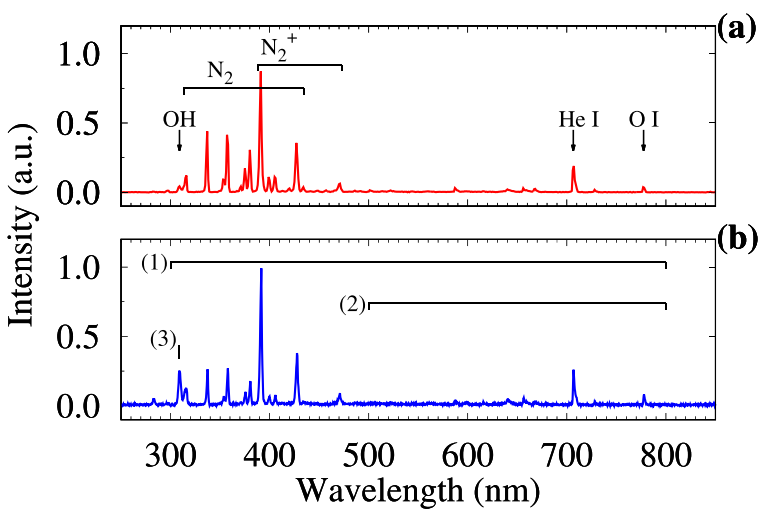

FIG. 2. APPJ integral spectrum: the sinusoidal voltage (a) and the superposition of bipolar square pulses and oscillating signals (b). Images (a1)-(f1) in Figs. 3 and 4 were obtained in the spectral range (1), (a2)-(f2) in (2), and (a3)-(f3) in (3). 

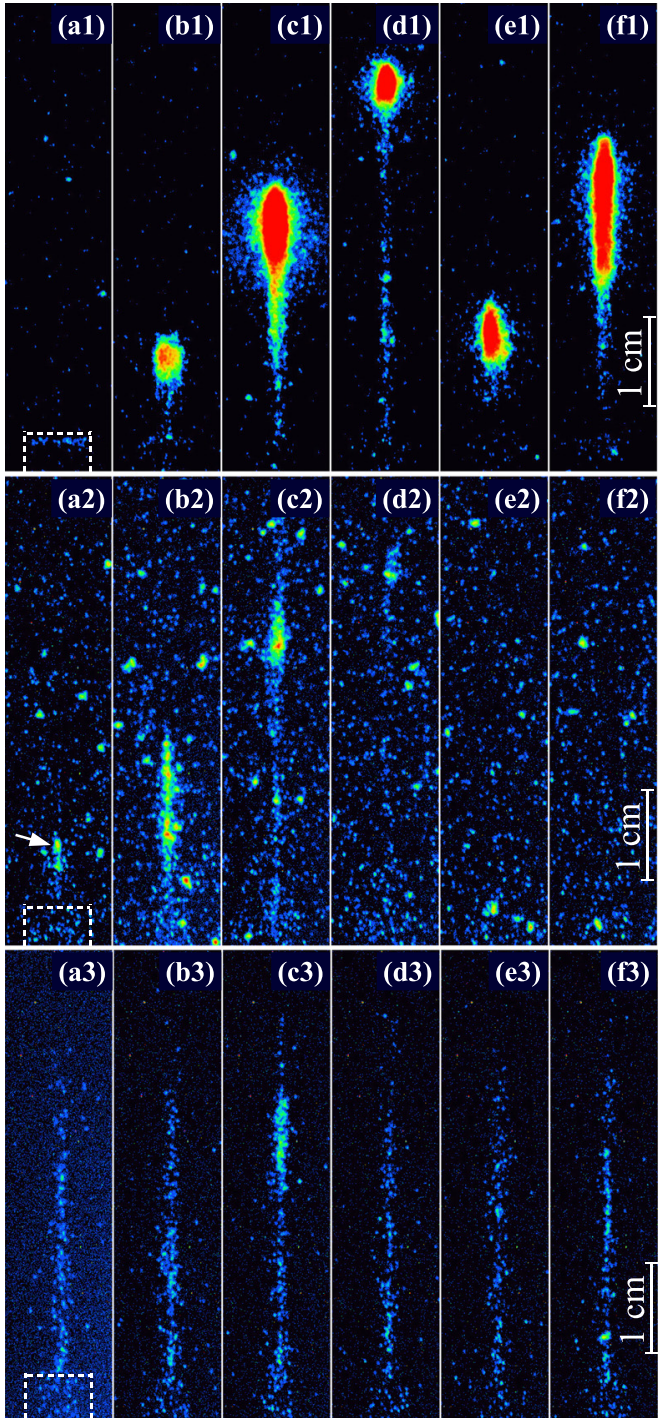

FIG. 3. ICCD images for the sinusoidal voltage in the spectral ranges of 300-800 nm (a1)-(f1), 500-800 nm (a2)-(f2), and $309 \mathrm{~nm}$ (a3)-(f3) for the time periods of $0 \mu \mathrm{s}(\mathrm{a}), 4.7 \mu \mathrm{s}(\mathrm{b}), 5.5 \mu \mathrm{s}(\mathrm{c}), 6.1 \mu \mathrm{s}(\mathrm{d}), 7.3 \mu \mathrm{s}(\mathrm{e})$, and $8.0 \mu \mathrm{s}(\mathrm{f})$. Dashed contours are the tube's borders.

emission; and imaging with an interference filter at $309 \mathrm{~nm}$ with a full width at half maximum of $10 \mathrm{~nm}$ [(a3)-(f3) in Figs. 3 and 4], which corresponded to $\mathrm{OH}$ radical emission. The number of accumulations was set to 50 for each image without a filter. Every image with optical filters was obtained via 150 accumulations due to the large loss of light in the filters.

Based on the image analysis, the APPJ lengths as functions of time for both voltages are plotted in Fig. 5. The position of the ionization front was defined as the boundary with a brightness that exceeded $50 \%$ of the maximum brightness in the images per voltage cycle.

The first observed discharge current peak in Fig. 1 at zero time for both voltage waveforms corresponds to the time when the voltage
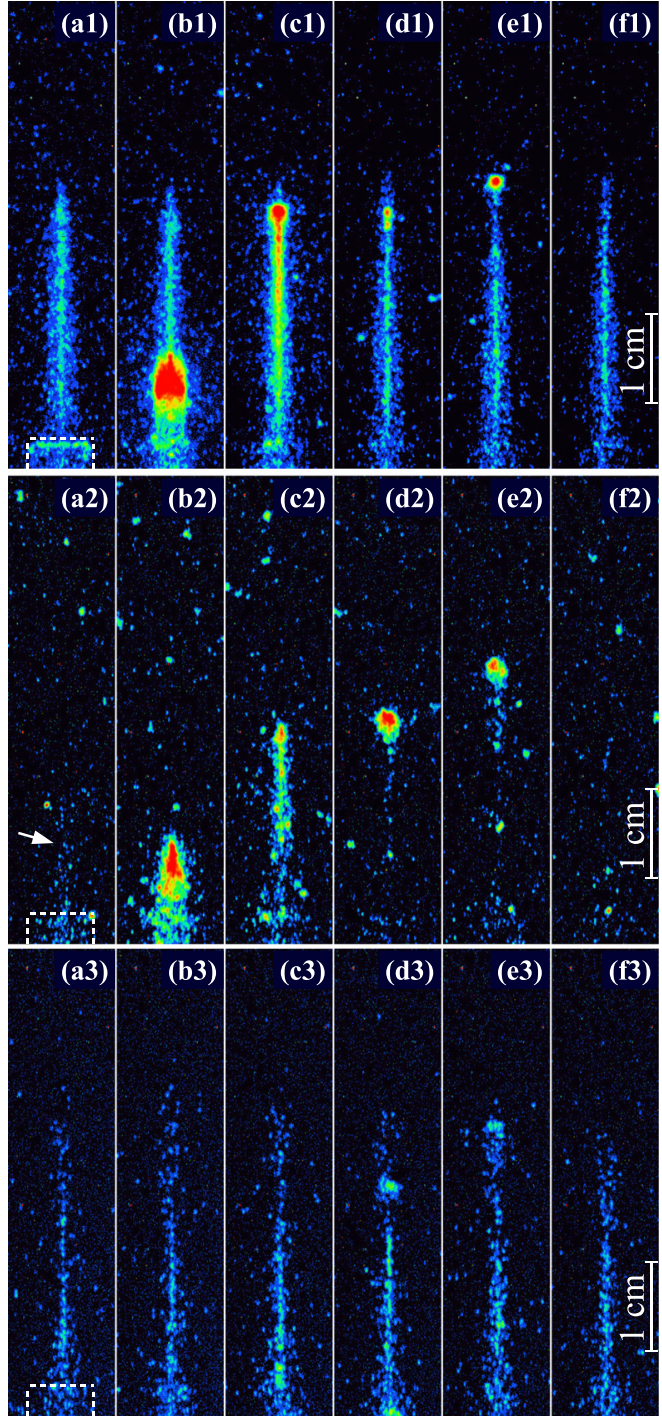

FIG. 4. ICCD images for the superposition voltage waveform in the spectral ranges of $300-800 \mathrm{~nm}(\mathrm{a} 1)-(\mathrm{f} 1), 500-800 \mathrm{~nm}(\mathrm{a} 2)-(\mathrm{f} 2)$, and $309 \mathrm{~nm}(\mathrm{a} 3)-(\mathrm{f} 3)$ for the time periods of $0 \mu \mathrm{s}(\mathrm{a}), 1 \mu \mathrm{s}(\mathrm{b}), 1.7 \mu \mathrm{s}(\mathrm{c}), 3.4 \mu \mathrm{s}(\mathrm{d}), 4.1 \mu \mathrm{s}(\mathrm{e})$, and $5.5 \mu \mathrm{s}(\mathrm{f})$. Dashed contours are the tube's borders.

reached the breakdown threshold value. A field enhancement near the tube end occurred after barrier charging, and the first IW started in the helium flow.

During the first current peak, a weak light emission zone with a short duration, which extended to a distance of approximately one centimeter, was observed [(a2) in Figs. 3 and 4, marked by arrows; red data points at zero time in Fig. 5]. Interestingly, the emission was defined almost completely by $\mathrm{He}$ I radiation at $706 \mathrm{~nm}$. We posit that this corresponds to a resonant photoexcitation of helium atoms that were generated from the radiation of the main discharge inside the tube, which is similar to the observed resonant reemission from the DBD microchannels that were detected in Ref. 29. 

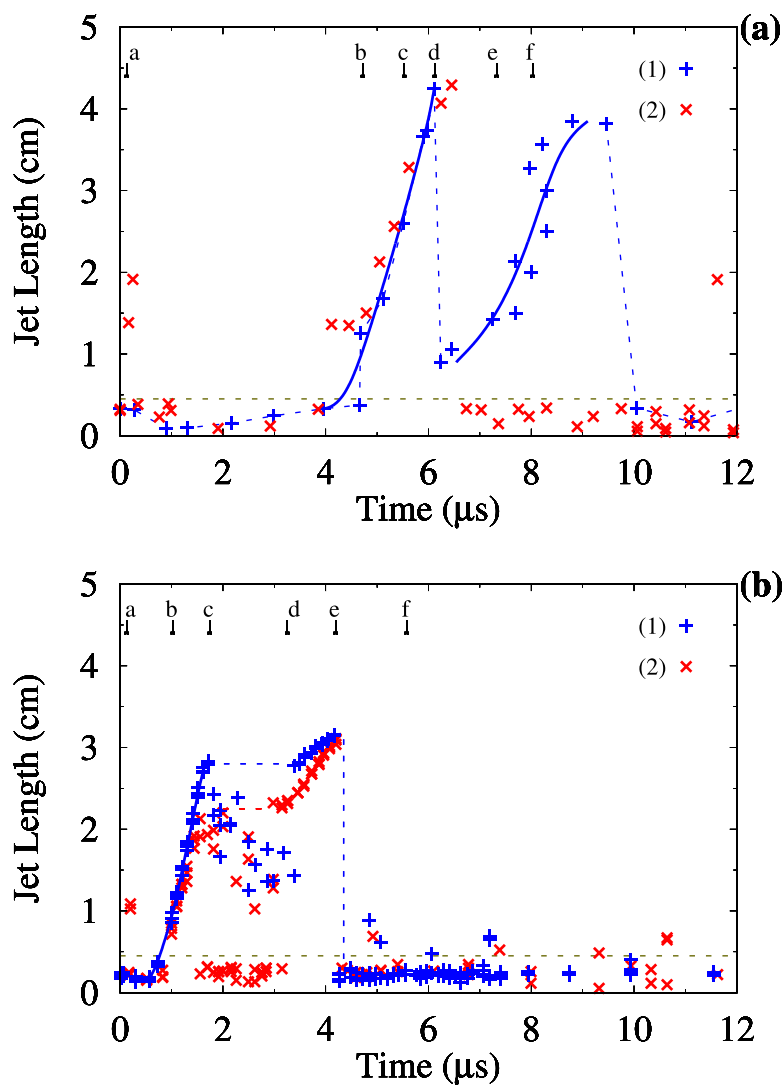

FIG. 5. APPJ length vs time: the sinusoidal voltage (a) and the superposition of bipolar square pulses and oscillating signals (b) in the spectral range of $300-800 \mathrm{~nm}$ (1) and estimated based only on He I emission (2). The labels (a)-(f) correspond to the frame labels in Figs. 3 and 4 . The horizontal dashed line shows the position of the tube orifice.

In the case of $\mathrm{OH}$ emission for both voltage regimes, an $\mathrm{OH}(\mathrm{X}-\mathrm{A})$ radiation was observed along the jet axis, mainly in the core of the effluent, (a3)-(f3) in Figs. 3 and 4. The level of radiation was almost constant along the jet and throughout the voltage period, even in the interval between the current pulses and the formation of the IWs. Hence, long-living excited $\mathrm{OH}$ radicals are present in the core of the jet all the time with a density that is almost independent of the position in the effluent. $\mathrm{OH}(\mathrm{A})$ species were produced due to the residual water content in the gas feeding system and are not related to the presence of water in the ambient air, which accords with Ref. 30. However, the observed phenomena do not contradict the proposed occurrence of the main production of $\mathrm{OH}$ radicals in the ground state at the APPJ boundary when mixed with air. ${ }^{31}$

In the case of the sinusoidal voltage, the plasma bullet dynamics differs completely from those initiated by the tailored voltage waveform. In the former case, the plasma bullet disappeared after it propagated to the end of the visible effluent; see (d1) in Fig. 3. A continuous increase in the voltage across the discharge gap resulted in the formation of a second bullet after approximately $3 \mu$ s past the first bullet; see (e1)-(f1) in Fig. 3. The ionization trace after the first ionization wave propagation enabled the second bullet to be sustained at a substantially smaller E-field than that was required for the generation of the first bullet. $^{32}$

The plasma properties in the second ionization wave differed from those of the first IW, as only radiation of molecular nitrogen bands was visible in the streamer head, and no He I emission was detected in the second IW. The intensity of the helium lines is a strong function of the electron temperature in the range of $2-10 \mathrm{eV}{ }^{33}$ The electron temperature in the same range is a function of the reduced electric field. ${ }^{34}$ According to Ref. 35, the emission intensity of the $\mathrm{He}$ $706.5 \mathrm{~nm}$ line is directly related to the electric field strength because the electric field has a strong effect on the electron temperature. This is well correlated with the low E-field that is required for second IW propagation and, correspondingly, the low electron temperature, which is not sufficient for He excitation. It is assumed that the field at the front of the second IW was of a strength of less than $5 \mathrm{kV} / \mathrm{cm}^{36}$ due to the intensity level of line detection, whereas for the first, the strength of the field was approximately $20 \mathrm{kV} / \mathrm{cm}$, according to Ref. 37 .

The slow rate of the voltage increase and decrease in the breakdown threshold led to a temporary jitter of the secondary bullet formation. This jitter resulted in scattering of experimental points for the APPJ length graph due to differences in terms of the front position among images for that time [blue points in the range of 7-9 $\mu \mathrm{s}$, Fig. 5(a)]. The blurring of image (f1) in Fig. 3 of the second bullet is due to this effect since the image was obtained by averaging over many shoots with a substantial jitter.

In the case of the tailored voltage waveform, the second voltage front in a positive half-period began at $\approx 2 \mu \mathrm{s}$ [Fig. 1(b)] and persisted until the channel conductivity was significantly reduced. ${ }^{38,39}$ Interestingly, the second plasma bullet is not formed in the jet but directly generated in the far effluent near the stagnation point of the first streamer head. The secondary IW amplified the field in the decaying plasma front after the first wave. The second IW is formed as the combined streamer continues to move [images (d) and (e) in Fig. 4]. This is a stepwise mode of the guided streamer propagation. ${ }^{24}$ The field at the front was of strength $10-20 \mathrm{kV} / \mathrm{cm}^{26,37}$

Much more intense radiation of He I in the streamer head was observed throughout the full time APPJ in the case of tailored voltage. This enabled us to conclude that the electron temperature was higher in this case. Moreover, the region with a high field is localized at a remote distance from the end of the dielectric tube and persists for a longer time than for the sinusoidal voltage.

The background weak emission persists in the APPJ for the entire period for which the jet is sustained by the tailored voltage; see (a1)-(f1) in Fig. 4. This is in contrast to the case of the sinusoidal voltage. Hence, excited species are present at a higher concentration in the case of the tailored voltage for the entire time period.

In summary, by changing the applied voltage shape, it was possible to localize the high-field region in space for a definite time, to change the plasma parameters in the guided streamer, and to change the radiative characteristics of the APPJ. Thus, voltage regime selection enables foreseeable changes to be made in the parameters of the APPJ.

The study was financially supported by the Russian Science Foundation (Project No. 18-79-10048).

The data that support the findings of this study are available within the article. And the additional data that support the findings 
of this study are available from the corresponding author upon reasonable request.

\section{REFERENCES}

${ }^{1}$ A. Fridman and G. Friedman, Plasma Medicine (Wiley, 2013).

${ }^{2}$ I. Adamovich, S. D. Baalrud, A. Bogaerts, P. J. Bruggeman, M. Cappelli, V. Colombo, U. Czarnetzki, U. Ebert, J. G. Eden, P. Favia, D. B. Graves, S. Hamaguchi, G. Hieftje, M. Hori, I. D. Kaganovich, U. Kortshagen, M. J. Kushner, N. J. Mason, S. Mazouffre, S. M. Thagard, H.-R. Metelmann, A. Mizuno, E. Moreau, A. B. Murphy, B. A. Niemira, G. S. Oehrlein, Z. L. Petrovic, L. C. Pitchford, Y.-K. Pu, S. Rauf, O. Sakai, S. Samukawa, S, Starikovskaia, J. Tennyson, K. Terashima, M. M. Turner, M. C. M. van de Sanden, and A. Vardelle, J. Phys. D: Appl. Phys. 50, 323001 (2017).

${ }^{3}$ S. Bekeschus, P. Favia, E. Robert, and T. von Woedtke, Plasma Processes Polym. 16, 1800033 (2019).

${ }^{4}$ A. Privat Maldonado, C. Bengtson, J. Razzokov, E. Smits, and A. Bogaerts, Cancers 11, 1920 (2019).

${ }^{5}$ X. Lu, M. Laroussi, and V. Puech, Plasma Sources Sci. Technol. 21, 034005 (2012).

${ }^{6}$ X. Lu, G. Naidis, M. Laroussi, and K. Ostrikov, Phys. Rep. 540, 123 (2014).

${ }^{7}$ X. Lu and K. K. Ostrikov, Appl. Phys. Rev. 5, 031102 (2018).

${ }^{8}$ M. Teschke, J. Kedzierski, E. G. Finantu-Dinu, D. Korzec, and J. Engemann, IEEE Trans. Plasma Sci. 33, 310 (2005).

${ }^{9}$ X. Lu, G. Naidis, M. Laroussi, S. Reuter, D. Graves, and K. Ostrikov, Phys. Rep. 630, 1 (2016).

${ }^{10}$ N. Y. Babaeva and G. V. Naidis, Trends Biotechnol. 36, 603 (2018).

${ }^{11}$ N. Y. Babaeva, N. Ning, D. B. Graves, and M. J. Kushner, J. Phys. D: Appl. Phys. 45, 115203 (2012).

${ }^{12}$ A. Shashurin, M. N. Shneider, and M. Keidar, Plasma Sources Sci. Technol. 21, 034006 (2012).

${ }^{13}$ A. Shashurin and M. Keidar, Phys. Plasmas 22, 122002 (2015).

${ }^{14}$ S. Iseni, C. Pichard, and A. Khacef, Appl. Phys. Lett. 115, 034102 (2019).

${ }^{15}$ B. Ghimire, E. J. Szili, P. Lamichhane, R. D. Short, J. S. Lim, P. Attri, K. Masur, K.-D. Weltmann, S.-H. Hong, and E. H. Choi, Appl. Phys. Lett. 114, 093701 (2019).

${ }^{16}$ G. V. Naidis and J. L. Walsh, J. Phys. D: Appl. Phys. 46, 095203 (2013).

${ }^{17}$ L. Lin and M. Keidar, Phys. Plasmas 23, 083529 (2016).
${ }^{18}$ Y. S. Akishev, V. B. Karalnik, M. A. Medvedev, A. V. Petryakov, N. I. Trushkin, and A. G. Shafikov, J. Phys.: Conf. Ser. 927, 012051 (2017).

${ }^{19}$ N. Benard and E. Moreau, Appl. Phys. Lett. 100, 193503 (2012).

${ }^{20}$ K. Gazeli, P. Svarnas, P. Vafeas, P. K. Papadopoulos, A. Gkelios, and F. Clément, J. Appl. Phys. 114, 103304 (2013).

${ }^{21}$ M. Pinchuk, A. Kudryavtsev, O. Stepanova, and D. Subbutin, in Proceedings of the VIII International Conference on Plasma Physics and Plasma Technology, PPPT-8 (2015), Vol. I, pp. 51-54.

${ }^{22}$ Y. Zhang, W. Ning, D. Dai, and Q. Wang, Plasma Sources Sci. Technol. 28, 104001 (2019).

${ }^{23}$ J. Wu, K. Wu, C. Ren, R. Liu, P. Jia, and X. Li, IEEE Trans. Plasma Sci. 47, 4868 (2019).

${ }^{24}$ M. E. Pinchuk, O. M. Stepanova, A. M. Astafiev, A. V. Lazukin, and Z. Chen, Appl. Phys. Lett. 114, 194103 (2019).

${ }^{25}$ O. Stepanova, M. Pinchuk, A. Astafiev, and Z. Chen, Jpn. J. Appl. Phys., Part 1 59, SHHC03 (2020).

${ }^{26}$ G. V. Naidis and N. Y. Babaeva, Phys. Plasmas 26, 094501 (2019).

${ }^{27}$ O. M. Stepanova, O. V. Rybalchenko, M. E. Pinchuk, A. M. Astafiev, O. G. Orlova, V. A. Spodobin, and A. A. Kudryavtsev, Plasma Med. 7, 187 (2017).

${ }^{28}$ M. Pinchuk, O. Stepanova, N. Kurakina, and V. Spodobin, J. Phys.: Conf. Ser. 830, 012060 (2017).

${ }^{29}$ V. Y. Khomich and M. V. Malashin, Plasma Processes Polymers 15, 1800053 (2018).

${ }^{30}$ R. P. Gott and K. G. Xu, IEEE Trans. Plasma Sci. 47, 4988 (2019).

${ }^{31}$ Y. Gorbanev, D. O’Connell, and V. Chechik, Chem.-A Eur. J. 22, 3496 (2016).

${ }^{32}$ N. L. Aleksandrov and E. M. Bazelyan, J. Exp. Theor. Phys. 91, 724 (2000).

${ }^{33}$ L. C. Johnson and E. Hinnov, Phys. Fluids 12, 1947 (1969).

${ }^{34}$ Y. P. Raizer, Gas Discharge Physics (Springer-Verlag Berlin Heidelberg, 1991).

${ }^{35}$ Q. Xiong, X. Lu, J. Liu, Y. Xian, Z. Xiong, F. Zou, C. Zou, W. Gong, J. Hu, K. Chen, X. Pei, Z. Jiang, and Y. Pan, J. Appl. Phys. 106, 083302 (2009).

${ }^{36}$ G. B. Sretenović, O. Guaitella, A. Sobota, I. B. Krstić, V. V. Kovačević, B. M. Obradović, and M. M. Kuraica, J. Appl. Phys. 121, 123304 (2017).

${ }^{37}$ G. B. Sretenović, I. B. Krstić, V. V. Kovačević, B. M. Obradović, and M. M. Kuraica, Appl. Phys. Lett. 99, 161502 (2011).

${ }^{38}$ X. P. Lu and M. Laroussi, Appl. Phys. Lett. 92, 051501 (2008).

${ }^{39}$ L. Nie, L. Chang, Y. Xian, and X. Lu, Phys. Plasmas 23, 093518 (2016). 